\title{
COMPARATIVE ACCURACY OF RADIOSTEREOMETRIC AND OPTICAL TRACKING SYSTEMS
}

\author{
Angela E. Kedgley, BASc, MESc *§ \\ Trevor Birmingham, $\mathrm{PhD}, \mathrm{PT} * \psi$ \\ Thomas R. Jenkyn, PhD, PEng * §\# \\ * Wolf Orthopaedic Biomechanics Lab \\ Fowler Kennedy Sport Medicine Clinic \\ London, Ontario, Canada N6A 3K7 \\ $\S$ Department of Mechanical and Materials Engineering \\ Faculty of Engineering \\ University of Western Ontario \\ London, Ontario, Canada N6A 5B9 \\ $\psi$ School of Physical Therapy \\ Faculty of Health Sciences \\ University of Western Ontario \\ London, Ontario, Canada N6G 1H1 \\ \# School of Kinesiology \\ Faculty of Health Sciences \\ University of Western Ontario \\ London, Ontario, Canada N6A 5B9
}

Correspondence:

Dr. Thomas Jenkyn

Department of Mechanical and Materials Engineering

Faculty of Engineering

University of Western Ontario

London, Ontario CANADA N6A 5B9

Phone: 519-661-2111 ext. 88339

Fax: 519-661-3020

Email: tjenkyn@uwo.ca

Keywords: fluoroscopy, RSA, motion analysis, accuracy, repeatability 


\section{Abstract}

This study aims to quantify and compare the accuracy of traditional radiostereometric analysis (RSA), fluoroscopic RSA (fRSA), and optical tracking systems. Three phantoms were constructed, each having three stainless steel spheres and three reflective markers. One phantom was mounted to the base of a precision cross-slide table, one to the base of a precision rotation table, and the third was mounted to each moveable tabletop. Two dial-gauges, rigidly mounted to the cross-slide table and rotation table, quantified translations and rotations. Two fluoroscopy units placed orthogonally tracked the steel spheres while a four-camera optical motion capture system tracked the reflective markers in three-dimensional space. RSA was performed with both digital radiography and fluoroscopy. Three axes of translation were tested: parallel to one fluoroscopy image, parallel to the other fluoroscopy image, and at approximately $45^{\circ}$ to each image. One axis of rotation was tested. Intraclass correlation coefficients indicated excellent agreement between the actual (dial-gauge) and measured translations for all modalities (ICCs $>0.99)$ and excellent agreement between actual and measured rotations for RSA and fRSA (ICCs $>0.99$ ). Standard errors of measurement ranged from $0.032 \mathrm{~mm}$ and $0.121^{\circ}$ for RSA, to $0.040 \mathrm{~mm}$ and $0.229^{\circ}$ for fRSA, and to $0.109 \mathrm{~mm}$ and $0.613^{\circ}$ for optical tracking. Differences between actual and measured translations along the $45^{\circ}$ axis were significantly smaller than the two parallel axes. These findings suggest that under ideal conditions, accuracy of fRSA is comparable to traditional RSA, and superior to optical tracking. Accuracy is highest when measured at $45^{\circ}$ to the fluoroscopy units. 


\section{Introduction}

Camera-based optical tracking systems are the most common method of quantifying three-dimensional joint kinematics. The direct linear transform algorithm reconstructs the three-dimensional locations of reflective or active markers placed on the skin over anatomic landmarks. Alternatively, bone pins topped with a cluster of reflective markers may be inserted into bones of interest, removing any potential for skin motion artefact. Radiostereometric analysis (RSA) provides an alternate method of quantifying joint positioning in-vivo. The algorithm for RSA is well-established in the literature (Selvik, 1989). Traditional RSA uses two static x-rays to provide simultaneous images of the object of interest from different angles. Often, the object has radio-opaque beads rigidly implanted to help determine its position. Using two views of the same markers, their positions are reconstructed into three-dimensional coordinates with accuracies on the order of \pm 10 microns (Madanat et al., 2005). The accuracy of measurements taken with traditional RSA using static digital images is about an order of magnitude better than conventional single-plane radiography (Ryd et al., 2000).

Recently, fluoroscopy and RSA have been integrated. Performing RSA with fluoroscopy rather than with static films is an appealing method to quantify joint kinematics in-vivo. It has the potential for accuracy surpassing that of optical tracking methods and approaching that of RSA with digital radiography. Work has been done previously to assess the accuracy, bias, and precision of RSA systems (Tashman and Anderst, 2003; Li et al., 2004; Ioppolo et al., 2007; Koning et al., 2007). Furthermore, one study used fRSA as the gold standard to quantify the accuracy of a skin-mounted tracking system (Garling et al., 2007). However, we are unaware of any previous 
research that has quantified and directly compared the measurement error for these three methods. The purposes of this study were to quantify and compare the accuracy of traditional RSA, fluoroscopic RSA (fRSA) and optical motion tracking. It was hypothesized that the differences between true translations and rotations and those measured with fRSA would be significantly smaller than those measured with optical tracking, but significantly greater than those measured using traditional RSA performed using digital radiography.

\section{Methods}

The fluoroscopic RSA system consisted of two fluoroscopy units (SIREMOBIL Compact (L), Siemens Medical Solutions USA Inc., Malvern, PA, USA) connected to two personal computers. A video capture device (DVD Xpress DX2, ADS Technologies Inc., Cerritos, CA) transferred images from the fluoroscopy units to the computer where the images were viewed and recorded by the accompanying software (Capture Wizard, ADS Technologies Inc., Cerritos, CA). Image processing software and the software to conduct the RSA analysis was written in MATLAB (The MathWorks, Natick, MA, USA). The system was calibrated with a custom-made calibration object similar to others previously described in the literature (Valstar et al., 2005). Fiducial planes consisted of 451 -mm-diameter beads, with a grid spacing of $20 \mathrm{~mm}$ by $25 \mathrm{~mm}$. Control planes consisted of 45 2-mm-diameter beads, with a grid spacing of $15 \mathrm{~mm}$ by $20 \mathrm{~mm}$.

Three phantoms, each consisting of three stainless steel spheres ( $1 \mathrm{~mm}$ diameter) were constructed. A cluster of three reflective markers was rigidly attached to each phantom. The locations of the reflective markers were tracked by a real-time motion analysis system in three-dimensional space using a four-camera optical motion analysis 
system (Hawk cameras, EvaRT system, Motion Analysis Corp., Santa Rosa, California, USA). The fluoroscopy units were positioned at right angles to one another, such that the phantoms could be seen in both images (Figure 1). Before tracking the phantoms, several images of the calibration object were collected to calibrate the RSA system. Translation and rotation were quantified.

To quantify translation, one of the phantoms was mounted to the base of a precision cross-slide table (Model VCT 514, Sowa Tool and Machine Co. Ltd., Kitchener, Ontario, Canada) to provide a fixed reference location throughout testing. A second phantom was mounted to the moveable tabletop. The applied translation was quantified by a dial-gauge rigidly mounted to the mechanism of the cross-slide table (IDS112, Mitutoyo Corporation, Kawasaki, Japan). The position of the tabletop was altered in increments of $0.25 \mathrm{~mm}$ and $0.50 \mathrm{~mm}$ along a single degree of freedom. The total distance traveled was $11.00 \mathrm{~mm}$. At each position, the locations of the fixed and moveable phantoms were quantified using digital radiography RSA, fRSA, and optical motion analysis. Three directions of translation were tested with respect to the fluoroscopes: parallel to fluoroscopy unit A, parallel to fluoroscopy unit B, and at approximately $45^{\circ}$ to each of the fluoroscopy units. The system was recalibrated prior to the testing along each axis.

To quantify rotation, the third phantom was mounted to the base of a precision rotation table (Model HV-6, Sowa Tool and Machine Co. Ltd., Kitchener, Ontario, Canada) to provide a fixed reference. The second phantom was again mounted rigidly to the table top. The applied rotation was quantified by a second dial-gauge that rigidly mounted to the rotation table. The table was rotated such that increments of $0.25 \mathrm{~mm}$ 
were read on the dial-gauge. This resulted in a rotation of $0.170^{\circ}$. The total distance travelled by the dial-gauge was $11.5 \mathrm{~mm}\left(7.70^{\circ}\right)$. Again, the locations of the fixed and moveable phantoms were quantified using RSA, fRSA, and optical motion analysis. One axis of rotation was tested.

For all cases, the positions of the beads within the fluoroscopy images were digitized and the three-dimensional positions of the beads were calculated using the standard RSA algorithm (Selvik, 1989). Distortion correction was performed on both the fluoroscopy images and the digital radiography images using a global approach by means of a fifth-order polynomial (Liu et al., 1999). To determine translation, for each phantom at each location, the centroid of the three beads and the centroid of the three reflective markers were determined. Translation was quantified as the change in distance between the reference phantom and the moveable phantom on the tabletop. To determine rotation angle, a coordinate system was created using the fixed phantom and the beads or markers on the moving phantom were transformed into the new, fixed frame of reference. Rotation was then quantified as the change in angle of a vector between any pair of the three points on the moving phantom.

The agreement between actual (dial-gauge) and measured translations and rotations was evaluated using intraclass correlation coefficients (ICC type 2,1) (Shrout and Fliess, 1979) and the error in individual translations was quantified using the standard error of measurement (Streiner and Norman, 1995). We also examined agreement using Bland and Altman plots of the difference between values against the mean of the values (Bland and Altman, 1986). The difference scores between the dialgauge and fRSA translations were then compared to those obtained with traditional RSA 
and optical tracking systems using paired t-tests. Lastly, difference scores obtained under different axes of translation were also compared with paired t-tests.

\section{Results}

During translation, the mean error of rigid body fitting for traditional RSA was $0.054 \pm 0.014 \mathrm{~mm}$ for the static phantom and $0.084 \pm 0.009 \mathrm{~mm}$ for the moving phantom, averaged over all axes of motion. Similarly, for fRSA the mean error of rigid body fitting was $0.129 \pm 0.009 \mathrm{~mm}$ for the static phantom and $0.109 \pm 0.0325 \mathrm{~mm}$ for the moving phantom. During rotation, the mean errors of rigid body fitting using traditional RSA were $0.067 \mathrm{~mm}$ and $0.172 \mathrm{~mm}$ for the static and moving phantoms, respectively. Using fRSA they were $0.134 \mathrm{~mm}$ and $0.161 \mathrm{~mm}$ for the static and moving phantoms, respectively.

The ICCs and standard errors of measurement for all measurement modalities and all axes of translation are listed in Table 1. Very good agreement was found between the actual and measured translations for all modalities with all ICCs being greater than 0.99 . Bland-Altman plots for the diagonal axis are shown in Figure 2. The difference scores between the dial-gauge and fRSA translations were not significantly different than those using traditional RSA $(p=0.469)$, but were significantly lower than those using the optical system $(\mathrm{p}<0.001)$. Using digital radiography, measured translations along the axis at $45^{\circ}$ to both fluoroscopy units were found to have significantly smaller errors than measured translations along either of the other two axes $(p=0.018$ and $p=0.021)$. Using fluoroscopy, measured translations along the $45^{\circ}$ axis were found to have significantly smaller errors than translations along the axis parallel to fluoroscopy unit $A(p=0.041)$. 
The ICCs and standard errors of measurement for rotation are listed in Table 2. Very good agreement was found between the actual and measured rotations for both RSA and fRSA (ICCs $>0.99)$, and to a lesser extent for the optical system $(\mathrm{ICC}=0.94)$. Bland-Altman plots for rotation are shown in Figure 3. The difference scores between the dial-gauge and fRSA translations were significantly different than those using digital radiography $(\mathrm{p}<0.001)$ with RSA resulting in smaller errors. Difference scores for both types of RSA were significantly lower than those of the optical system $(p<0.001)$.

\section{Discussion}

This study has shown that images taken using fluoroscopy under ideal conditions - in this case a phantom - can produce RSA reconstructions that are as accurate as those taken with digital radiography. Results also show that fRSA is significantly more accurate in tracking a rigid body than an optical tracking system. These results suggest that, if feasible, the insertion of tantalum beads to track kinematics is likely more accurate than the use of reflective markers, even if these are attached to bone pins. Furthermore, examination of the results from the three translational axes of motion suggests that the main plane of interest should be positioned such that it lies at $45^{\circ}$ to both fluoroscopy units in order to take advantage of the increased accuracy.

Since fluoroscopic images may be taken while the subject is in motion, comparable accuracy for RSA obtained with fluoroscopy and digital radiography suggests that fRSA should enable substantial improvements to the study of even very subtle in-vivo kinematics. The importance of studying dynamic activities should be acknowledged, because when joints are in motion the inertias of the body segments are incorporated, thereby more realistically replicating the activities of daily living. 
However, a major drawback with fluoroscopy is the blurring that occurs when motion is too quick. Additional research is, therefore, needed to determine how the accuracy of fRSA changes with increasing object velocities. It should also be noted that fRSA (and RSA using digital radiography taken with fluoroscopy units) has the added disadvantage of a small capture volume - generally limited to one joint. The combination of fRSA with an optical tracking system may provide the solution to this dilemma.

While optical motion analysis proved to be less accurate than RSA, the accuracies attained were nonetheless very good and it should certainly still be considered an excellent method for rigid body tracking. Clearly there are many situations where the use of optical surface markers is more appropriate for the study of kinematics than the implantation of tantalum beads. Practically, the greatest problem with the use of reflective markers is the error that arises in the rigid body assumption when markers are attached to the skin. Optical motion analysis accuracy may also be influenced by the placement of the tracking cameras. Although it is possible that the accuracies obtained using the optical system in this study may have been improved had the cameras been placed closer to the translation table, shrinking the capture volume, we felt that it was important to mimic the conditions most often used for in-vivo, functional testing. Also, additional care must be taken when processing data from optical motion analysis systems. For example, if aberrant trials had not been detected during post-processing in the present study, accuracy results would have dropped dramatically $\left(\mathrm{ICC}=0.53, \mathrm{SEM}=1.72^{\circ}\right)$. Ultimately, it is the clinical question being addressed by a study and the accuracy that is required of the results that will determine which modality is best for examining threedimensional kinematics. 
In conclusion, the present findings suggest that under ideal conditions, accuracy

of fRSA is comparable to RSA, greater than optical tracking and highest when measured at $45^{\circ}$ to the fluoroscopy units.

\section{Acknowledgements}

The authors would like to thank Ms. Anne Marie Allen and Mr. Adam Crymble for their assistance with data collection and the Natural Sciences and Engineering Research Council for their financial support.

\section{References}

Bland, J.M., Altman, D.G., 1986. Statistical methods for assessing agreement between two methods of clinical measurement. Lancet 1, 307-310.

Garling, E.H., Kaptein, B.L., Mertens, B., Barendregt, W., Veeger, H.E.J., Nelissen, R.G.H.H., Valstar, E.R., 2007. Soft-tissue artifact assessment during step-up using fluoroscopy and skin-mounted markers. Journal of Biomechanics 40, S18-S24.

Ioppolo, J., Börlin, N., Bragdon, C., Li, M., Price, R., Wood, D., Malchau, H., Nivbrant, B., 2007. Validation of a low-dose hybrid RSA and fluoroscopy technique:

Determination of accuracy, bias and precision. Journal of Biomechanics 40, 686-692.

Koning, O.H.J., Kaptein, B.L., Garling, E.H., Hinnen, J.W., Hamming, J.F., Valstar, E.R., van Bockel, J.H., 2007. Assessment of three-dimensional stent-graft dynamics by using fluoroscopic roentgenographic stereophotogrammetric analysis. Journal of Vascular Surgery $46,773-779$.

Li, G., Wuerz, T.H., Defrate, L.E., 2004. Feasibility of using orthogonal fluoroscopic images to measure in vivo joint kinematics. Journal of Biomechanical Engineering 126, 314-318.

Liu, R.R., Rudin, S., Bednarek, D.R., 1999. Super-global distortion correction for a rotational C-arm X-ray image intensifier. Medical Physics 26, 1802-1810.

Madanat, R., Makinen, T.J., Moritz, N., Mattila, K.T., Aro, H.T., 2005. Accuracy and precision of radiostereometric analysis in the measurement of three-dimensional micromotion in a fracture model of the distal radius. Journal of Orthopaedic Research 23, 481-488.

Ryd, L., Yuan, X., Lofgren, H., 2000. Methods for determining the accuracy of radiostereometric analysis (RSA). Acta Orthopaedica Scandinavica 71, 403-408. 
Selvik, G., 1989. Roentgen stereophotogrammetry. A method for the study of the kinematics of the skeletal system. Acta Orthopaedica Scandinavica Supplement 232, 151.

Shrout, P.E., Fleiss, J.L., 1979. Intraclass Correlations: Uses in Assessing Rater Reliability. Psychological Bulletin 86, 420-428.

Streiner, D.L., Norman, G.R., 1995. Health Measurement Scales: A Practical Guide to their Development and Use. Oxford University Press, New York.

Tashman, S., Anderst, W., 2003. In-vivo measurement of dynamic joint motion using high speed biplane radiography and CT: Application to canine ACL deficiency. Journal of Biomechanical Engineering 125, 238-245.

Valstar, E.R., Gill, R., Ryd, L., Flivik, G., Börlin, N., Kärrholm, J., 2005. Guidelines for standardization of radiostereometry (RSA) of implants. Acta Orthopaedica. 76 (4), 563 572.

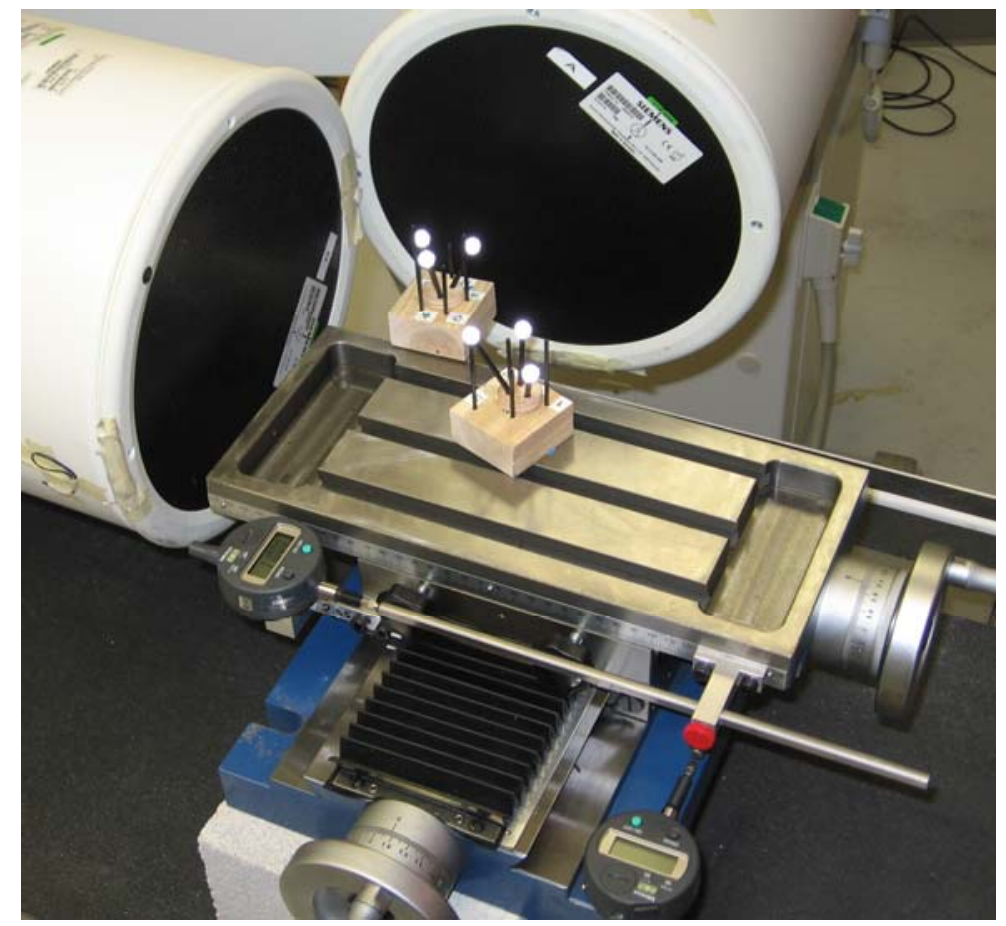

Figure 1. Translation experimental setup. The two fluoroscopy units were placed at approximately right angles to one another. One phantom was mounted to the base of a precision cross-slide table and a second phantom was mounted to the moveable top of the table. The applied translation was quantified by a dial-gauge rigidly mounted to the mechanism of the crossslide table (seen at bottom-left of the photograph). 

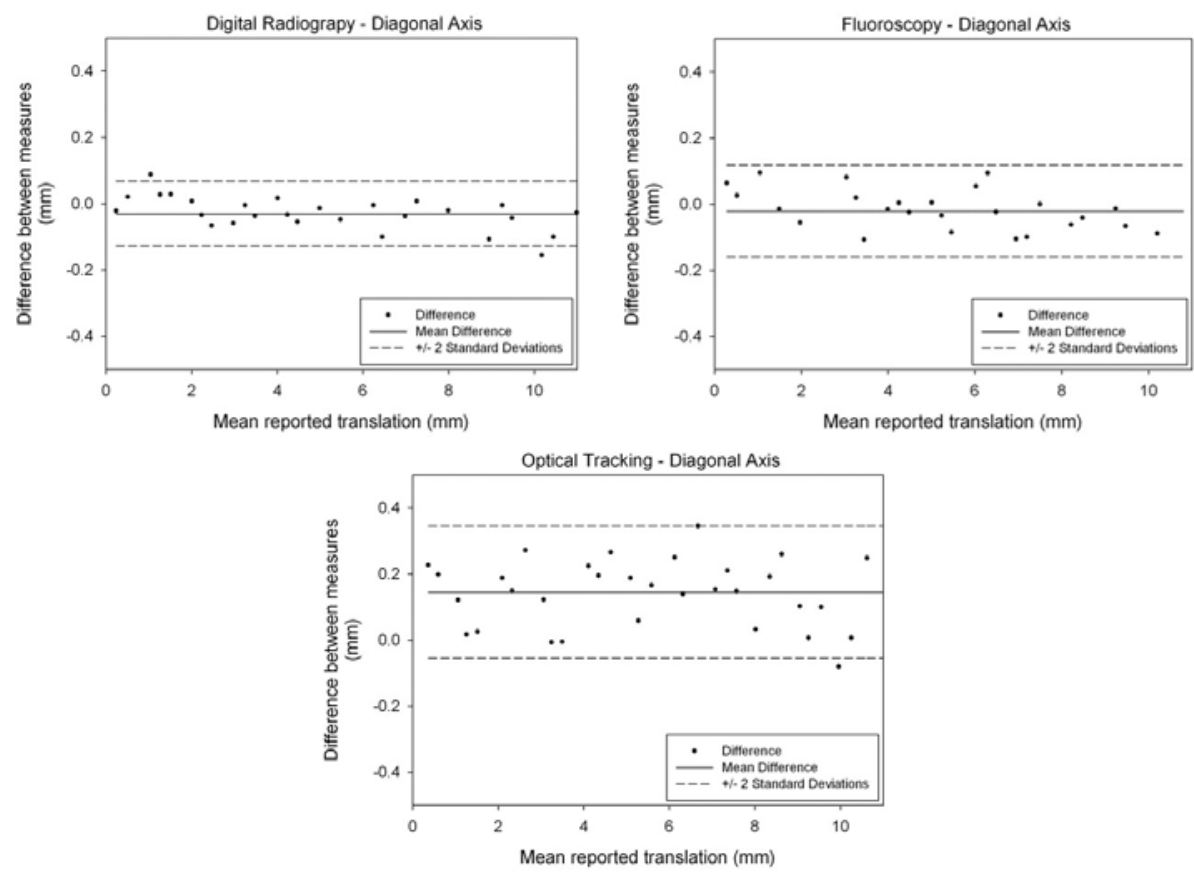

Figure 2. Bland-Altman plots for the three imaging modalities during translation: Digital radiography, fluoroscopy and optical motion analysis for translation along the diagonal axis of motion. The horizontal axis in all plots is the mean translation of the two measures (dial-gauge versus the particular imaging modality) and the vertical axis is the difference between the two measures.
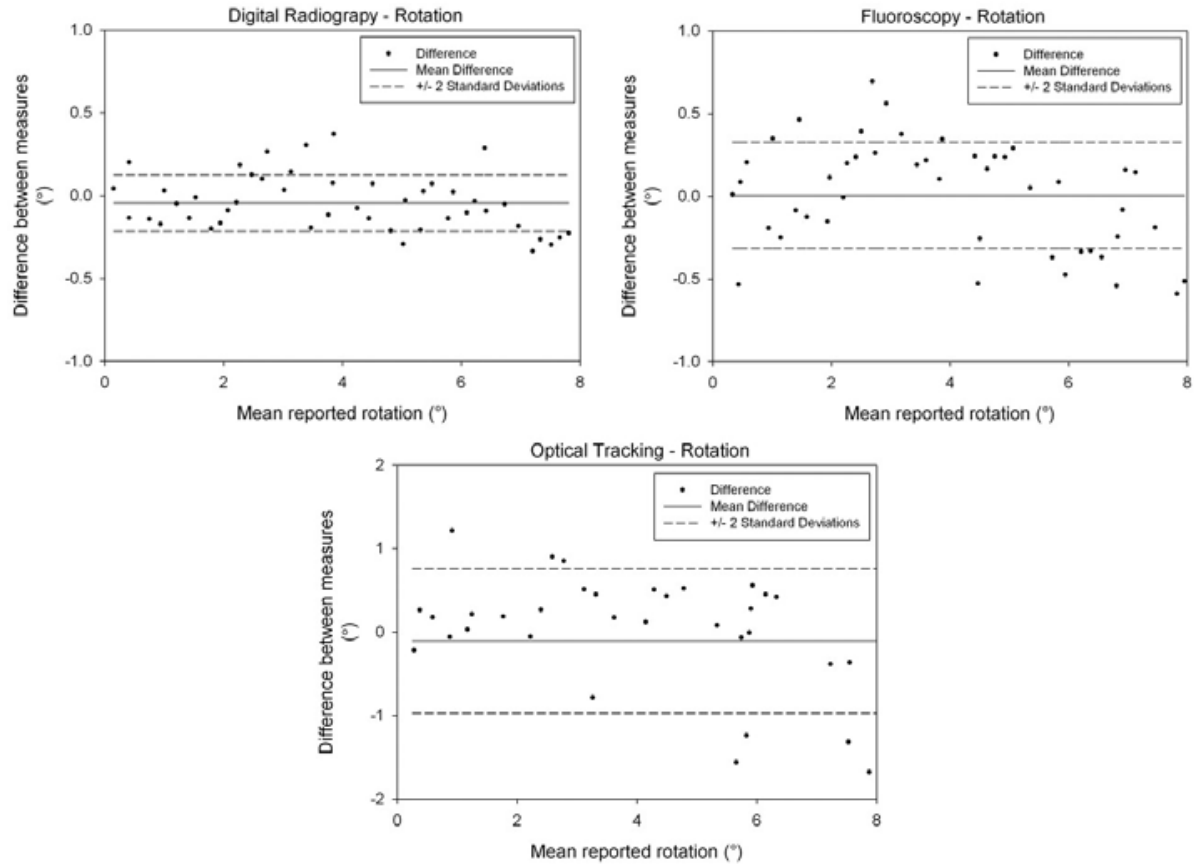

Figure 3. Bland-Altman plots for the three imaging modalities during rotation: Digital radiography, fluoroscopy and optical motion analysis. The horizontal axis in all plots is the mean rotation of the two measures (dial-gauge versus the particular imaging modality) and the vertical axis is the difference between the two measures. 
Table 1. Intra-class correlation coefficients (ICCs) and standard errors of measurement for all three imaging modalities and all three axes of translation tested.

\begin{tabular}{|l|c|c|c|}
\hline \multicolumn{1}{|c|}{ Imaging Mode } & Axis & ICC & $\begin{array}{c}\text { Standard Error } \\
\text { of Measurement } \\
(\mathbf{m m})\end{array}$ \\
\hline Digital radiography & Diagonal & 0.9999 & 0.036 \\
\hline Digital radiography & Parallel to A & 0.9996 & 0.032 \\
\hline Digital radiography & Parallel to B & 0.9997 & 0.039 \\
\hline Fluoroscopy & Diagonal & 0.9998 & 0.043 \\
\hline Fluoroscopy & Parallel to A & 0.9995 & 0.040 \\
\hline Fluoroscopy & Parallel to B & 0.9998 & 0.050 \\
\hline Optical & Diagonal & 0.9986 & 0.071 \\
\hline Optical & Parallel to A & 0.9951 & 0.109 \\
\hline Optical & Parallel to B & 0.9994 & 0.069 \\
\hline
\end{tabular}

Table 2. Intra-class correlation coefficients (ICCs) and standard errors of measurement using all three imaging modalities for rotation.

\begin{tabular}{|l|c|c|}
\hline \multicolumn{1}{|c|}{ Imaging Mode } & ICC & $\begin{array}{c}\text { Standard Error } \\
\text { of Measurement } \\
\mathbf{(}^{\circ}\end{array}$ \\
\hline Digital radiography & 0.9970 & 0.121 \\
\hline Fluoroscopy & 0.9904 & 0.229 \\
\hline Optical & 0.9386 & 0.613 \\
\hline
\end{tabular}

